\title{
EL LUGAR DE LA CULTURA Y LA CULTURA DEL LUGAR: PRÁCTICAS Y CONOCIMIENTOS DE LOS CRIADORES DE CAMÉLIDOS EN EL SUR PERUANO
}

\author{
THE PLACE OF CULTURE AND THE CULTURE OF PLACE: \\ PRACTICES AND KNOWLEDGE OF CAMELID BREEDERS \\ FROM SOUTHERN PERU
}

\author{
Percy Arrosquipa ${ }^{1}$
}

\begin{abstract}
Para analizar la importancia de la cultura local en procesos de modernización y desarrollo local, se estudiaron las prácticas y conocimientos que caracterizan el proceso de crianza y comercialización realizada por criadores de camélidos en la provincia de Caylloma, Perú, a través de cinco estudios de casos, uno por distrito o anexo. Los resultados muestran que la crianza de camélidos domésticos se caracteriza por ser rotatoria y trashumante, por ser un proceso holístico donde converge la comunidad, la naturaleza y lo sobrenatural, expresado en creencias, prácticas y rituales, que es soslayada en procesos de desarrollo. La crianza de camélidos y comercialización de fibra, es practicada con participación familiar y a menudo en asociatividad con vecinos de la estancia, con evidente liderazgo de la mujer. Entre los sistemas de comercialización practicada por criadores tenemos la individual a través de intermediarios y la asociada a través de centros de acopio. La comercialización asociada es un modelo arraigado a la cooperación familiar y asociatividad practicada en la cultura local, que implica participar en estrategias colectivas. Esto demuestra que las prácticas y conocimientos locales tienen viabilidad y son esenciales en procesos de modernización local, cuya sostenibilidad se basa en la diversidad cultural y ecológica.
\end{abstract}

Palabras claves: cultura, camélidos, crianza, comercialización, desarrollo, conocimientos locales.

In order to analyze the importance of local culture in the processes of modernization and local development, we researched the knowledge and practices that typify the breeding and commercialization process employed by breeders of camelids in Caylloma province, Peru, through five case studies, one for each town or district. Our results indicate that breeding of domestic camelids is a rotational and migratory activity, as well as a holistic process which brings together the community, nature and even the supernatural, which is manifested in beliefs, practices and rituals that are usually overlooked in development processes. The breeding of camelids and the commercialization of their fiber involve the participation of the entire family, often with the collaboration of neighbours of the grazing territory, and openly conducted under the leadership of the women. Among the systems of commercialization used by breeders, we observed: the individual through intermediaries, and partnership through storage centers. The partnership system is a model based on family cooperation and principles of strategic collaboration as carried out in the local culture, which involve participating in collective strategies. This demonstrates that the practices and local knowledge are viable and essential for processes of local modernization, the sustainability of which is based on cultural and ecological diversity.

Key words: Culture, camelids, breeding, commercialization, development, local knowledge.

\section{Perspectiva Teórica: el Lugar de la Cultura y la Cultura del Lugar}

La actividad económica que predomina en la parte alta del valle del Colca (Figura 1) es la crianza de camélidos sudamericanos domésticos, preferentemente alpacas y en poca proporción llamas, fuente de seguridad alimentaria y generación de ingresos para los criadores de camélidos domésticos (Markowitz 2006). Esta actividad económica implica un estilo de comportamiento, prácticas y conocimientos, pero en acciones dirigidas a superar la pobreza en comunidades rurales del Perú, normalmente ha merecido poca o ninguna atención, al entender el desarrollo como imposición (exógeno). El hombre andino, considerando sus concepciones y recursos de su entorno, desarrolló conocimientos y tecnologías de base ecológica e impregnadas de rituales, un recurso cultural vinculado a su cosmovisión, que expresa su pensamiento y concepción de su cotidianidad, su entorno natural y cultural (García 1996). Como sostiene Geertz (1992:118-125) "la cosmovisión es su retrato de la manera en que las cosas son en su pura efectividad, es su concepción

$\overline{1}$ Universidad Nacional de San Agustín, Arequipa, Perú. aqpercy@yahoo.com 


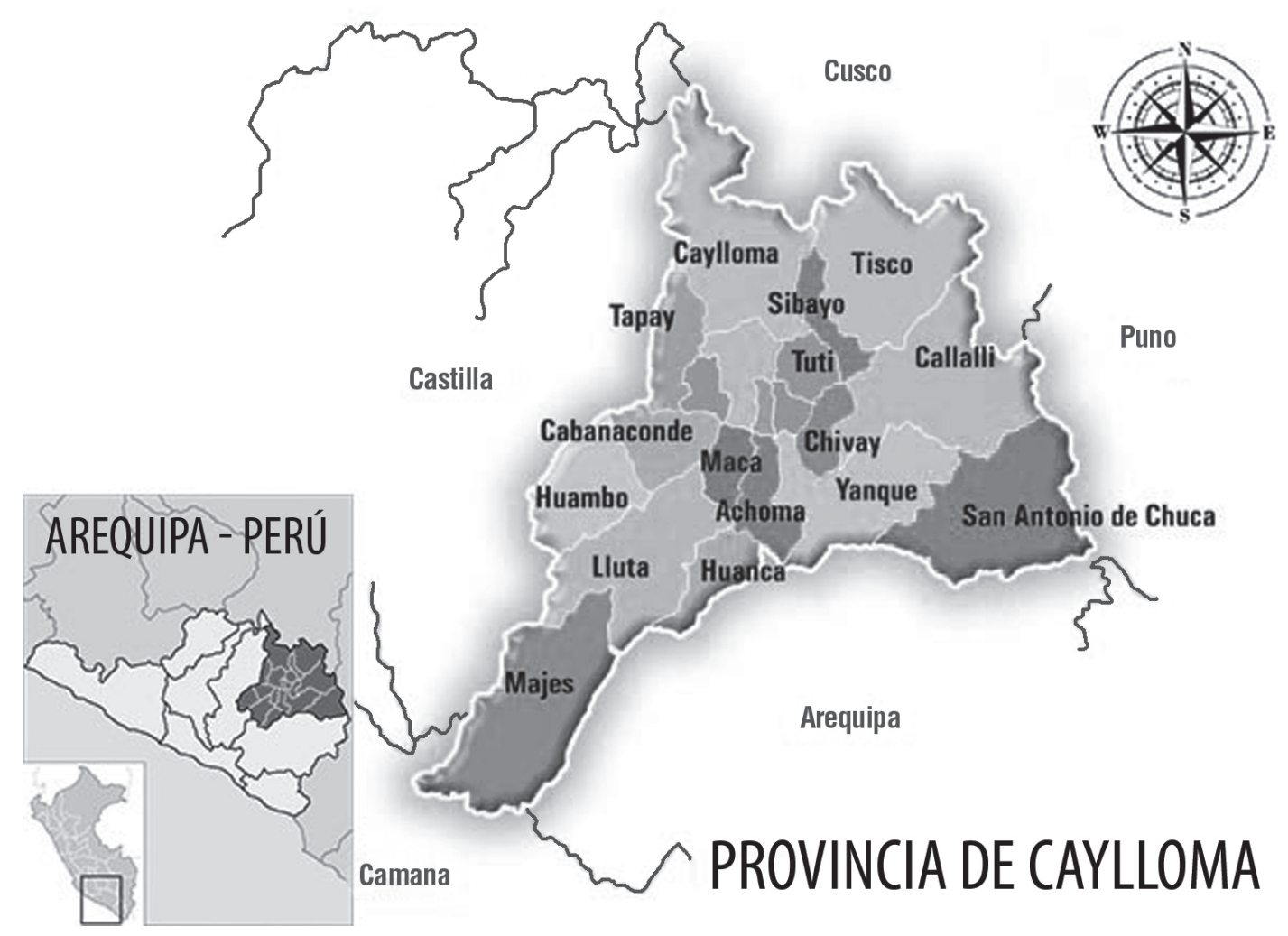

Figura 1. Crianza de camélidos domésticos en distritos de la zona alta. Breeding of domestic camelids in the highland districts.

de la naturaleza, de la persona, de la sociedad". La cosmovisión contiene las ideas más generales sobre diversos aspectos de la vida en una comunidad, y varía en el tiempo, de una cultura a otra. Lo que un pueblo valora, cree, desea, odia está pintado en su cosmovisión, simbolizados en su cultura y representados en su estilo de vida y/o ethos, que la distingue no solo desde cómo conceptualiza la excelencia y lo bueno, sino también desde el punto de vista con que condena y desaprueba. En esa perspectiva, un comportamiento poco ético, una innovación tecnológica incompatible, una costumbre poco usual, sería inviable, no tendría razón de ser, carecería de naturalidad y de simple efectividad.

Algunas culturas valoran positivamente el cambio y la novedad, en otras culturas ejerce una atracción menos positiva. Conocer la cultura de un grupo étnico viabiliza una innovación culturalmente adecuada, pero no suele ser una constante en programas de mejoramiento ni en procesos de innovación tecnológica, que encontraron dificultades y fracasos por no haber reconocido previamente formas culturales definidas (Foster 1976). Aquí, el dilema entre la tradición y la modernidad es intenso, a veces incompatible, dado que la modernidad critica a la tradición al atribuirle un carácter normativo, que pone barreras al cambio. Tal atribución responde al discurso filosófico de la modernidad de occidente (racionalidad burocrática), que desconoce la modernidad y racionalidad del espacio simbólico de los pueblos (Urbano 1991). Entonces, resulta distinto reconocer el carácter histórico de la tradición y muy distinto es atribuirles a la historia y a la tradición un carácter normativo. La historia y la tradición no es lo que está en juego con la "llegada" de la modernidad, de lo contrario no sería posible la modernidad de pueblos orientales [como Japón, China e India] arraigados a su cultura.

Pero promover el desarrollo local sostenible, mejorar la eficacia de intervenciones sociales, introducir tecnologías [modernas], no es problema de tractores, maquinarias, insumos. Con chakitaqlla o tractor, con zapatos o sandalias, los hombres y mujeres de los Andes pueden ser modernos, 
anticuados o posmodernos. La situación es qué función cumple en la actualidad y por qué perviven hasta nuestros días las prácticas y conocimientos locales, la cooperación familiar y la asociatividad comunitaria, sustentada en la reciprocidad (ayni), trabajo comunitario (minka) y redes comunales o de compadrazgo, cuyo fortalecimiento es imprescindible para mejorar la capacidad productiva y de negociación de los criadores de camélidos, por ende su participación equitativa en el mercado. Entonces, el retorno o presencia de lo antiguo en la sociedad contemporánea, no son obstáculo para la modernidad local. Las instituciones u organizaciones tradicionales pueden ser reorientadas y expresar un nuevo campo semántico dentro de experiencias sociales, nuevas, muy diferentes a aquellos en que nacieron.

Las formas de organización y desarrollo tecnológico son mecanismos de adaptación sociocultural, guardan relación con el complejo mundo ideológico y principios cosmológicos, presente en su organización económica, política y social. Por ejemplo, en el mundo andino la división del trabajo [para la mujer] no está en función de la fuerza física, sino en función del tiempo y espacio (Flores 1977). Las formas de organización, conocimiento y paradigmas que caracterizan a la cultura local evidencian que la crianza de alpacas y la comercialización de fibra no solo es explotación y aprovechamiento de recursos de un piso ecológico de los Andes, es también un complejo mundo ideológico (natural y sobrenatural), que prescribe pautas para el pastoreo, comportamiento, uso de tecnologías, organización, comercialización, entre otros.

El estudio realizado por Kevin Healy (2001, reseñado por Foley 2002) expone cómo la población indígena y algunos aliados revolucionaron la forma de pensar respecto de la herencia cultural y prácticas agrícolas en Bolivia, aun cuando administradores de agencias externas del occidente, junto a prejuicios de planificadores, agrónomos, zootecnistas y especialistas del desarrollo, pensaron que la cultura y las organizaciones indígenas eran obstáculo para el desarrollo. Es decir, las prácticas agrícolas, ganaderas y alimentarias indígenas, no solo significaban atraso, sino estaban destinados a desaparecer con la modernidad. El trabajo de Healy documenta procesos de mejoramiento de rebaño de llamas y alpacas, la recuperación del arte de tejer, casi perdido entre los Jalq'a de la zona central de Bolivia, relata el descubrimiento y comercialización de artesanías, extensión de educación a las mujeres en las serranías desoladas de Oruro, recuperación del vasto conocimiento indígena y otros aspectos. Eso evidencia que las destrezas de organización propia y los conocimientos locales son elementos claves para el desarrollo participativo y sostenible.

En el Perú se priorizaron aspectos técnicos y económicos en la lucha contra la pobreza en comunidades campesinas, dejando de lado la cultura del productor rural. Esa desatención a prácticas y conocimientos locales que perviven, explica los pésimos resultados del proceso de modernización impuesto en el mundo rural, alentado por modelos de desarrollo rural convencionales, como desarrollo rural "planificado" o "inducido" y desarrollo "integrado" o "unificado" (Van Der Ploeg 2000), que introdujo los "tipos ideales" mejorados, superiores en rendimiento, dado el uso de agroquímicos, pero no en variedad ni en calidad. Esto amerita que la cultura local no solo debe ser estudiada y comprendida, sino considerada e integrada como base fundamental en procesos de desarrollo local. Es decir, en aquella modernización que haga visible la diversidad cultural y ecológica, y no su homogeneización o estandarización.

En la actualidad existe la necesidad de implementar modelos de desarrollo más sensibles con las poblaciones locales y con el medioambiente, en esta tendencia aparecen: (a) el desarrollo sostenible o ecológico (Baigorri 2001), (b) desarrollo local participativo, (c) desarrollo propio (endógeno), (d) etnodesarrollo y otras variantes ${ }^{1}$. Estos modelos retoman la diversidad "tradicional" y la producción ecológica, que la perspectiva del posdesarrollo busca hacer visible. En este contexto, los conocimientos y prácticas locales tienen un lugar fundamental en procesos de desarrollo socioeconómicos modernos, entendidos como la capacidad de identificar y discutir la solución de sus problemas desde la propia visión. El desarrollo tiene que ver, menos con lo económico y más con la capacidad de las personas y los factores sociales y culturales (Escobar 1997), cuya sostenibilidad halla sus raíces en prácticas y discursos alternativos sobre la diversidad cultural y ecológica. En la perspectiva del posdesarrollo el proceso de modernización local con énfasis en la diversidad cultural y ecológica tiene un lugar fundamental, es lo que se denomina posdesarrollo ${ }^{2}$, que se contrapone al discurso universalista y homogeneizante del desarrollo convencional, con énfasis en el contexto del lugar, entendida como cultura local, como una forma de representación y concepción (Escobar 2000). El posdesarrollo busca la diversidad local, 
frente al desarrollo homogeneizador, el que desde su perspectiva monocultural concibe a las tradiciones y prácticas locales como obstáculos, y en el cual, los agricultores, ganaderos y pueblos tradicionales aparecen como "atrasados".

Si el propósito del posdesarrollo es hacer visible prácticas y conocimientos locales alternativos ecológica y culturalmente en un contexto global, implica un proceso de mejoramiento basado en el lugar de la cultura y la cultura del lugar, es decir, basado en conocimientos y prácticas locales, que perviven o se proyectan globalmente en defensa de los lugares y la identidad, por otro lado, los conocimientos y prácticas globales que los locales pueden utilizar para sus propios fines. Por eso, es importante tomar en cuenta la reconsideración de lo local y lo global en las políticas de desarrollo y en procesos de modernización local, para mejorar las prácticas y conocimientos locales sobre la comercialización de fibra y crianza de camélidos domésticos.

Bajo la denominación de camélidos sudamericanos se engloba dos especies salvajes: guanaco (Lama guanicoe) y vicuña (Lama vicugna), y dos especies domésticas: llama (Lama glama) y alpaca (Lama pacos). La mayoría de casos de crianza de camélidos se encuentra sobre los $3.500 \mathrm{msm}$. Históricamente en el incanato su distribución era amplia, la reproducción de camélidos era controlada a través de Kipus, para asegurar la pureza y calidad de la especie, 100 años después de la invasión española en 1532, el ecosistema andino cambió y fueron diezmadas las llamas y alpacas, teniendo como consecuencia un empobrecimiento genético por hibridación de ambas especies (Wheller 1991:37). Actualmente se sigue practicando la crianza de llamas por su capacidad de carga y la alpaca por su carne y fibra, siendo la crianza de camélidos domésticos una actividad económica importante, donde la comercialización de productos o subproductos derivados de la crianza de camélidos permite a los criadores tener seguridad alimentaria y generación de ingresos económicos. Desde luego, a mediados del siglo XIX, los criadores de la provincia Caylloma participan en circuitos globales de comercialización, con la explotación de fibra, proceso comercial que articula organizaciones, actores locales, intermediarios y empresas.

\section{Material y Métodos}

El estudio de casos con criadores de camélidos ${ }^{3}$ se realizó en la provincia de Caylloma, departamento de Arequipa, al sur del Perú a 3.630 msm. Caylloma tiene una población de 73.718 habitantes (INEI 2007), dedicada a la actividad agrícola y ganadera, del cual 2.541 familias se dedican a la crianza de alpacas (FAO 2008). La zona agrícola comprende desde 2.300 hasta $3.800 \mathrm{msm}$; y desde 3.800 hasta 4.800 msm comprende la zona ganadera (DESCO 1985), principalmente de camélidos domésticos, ubicada en la cuenca alta del Colca, donde se realizaron las entrevistas y la observación participante, teniendo como instrumentos: guía de entrevista y diario de observación de campo, una metodología etnográfica a través de estudio de casos, con participación vivencial en el proceso de crianza de alpacas y comercialización de fibra practicada por criadores. La selección de informantes se realizó a través de la observación participativa, que permitió conocer en el lugar a criadores de camélidos cualificados para el estudio (unidad de estudio), elegidos según los siguientes criterios: personas de mayor experiencia dedicados a la crianza de camélidos, participación en transacciones comerciales de ferias locales y, que vivieran en el ámbito de estudio del proyecto (Figura 1).

Se trabajó con cinco casos, teniendo un caso por distrito y/o anexo. El primero procedía de la parcialidad Huarasayco, anexo Chichas (distrito Callalli), el segundo y tercero de Chalhuanca (distrito Yanque), el cuarto de Comunidad Caylloma (distrito Caylloma) y quinto de Comunidad Ran Ran (distrito Tuti). Con ellos se procedió a conversar, entrevistar y participar en sus actividades cotidianas de crianza y comercialización. En ese contexto se realizaron visitas de trabajo a las estancias ${ }^{4}$ para realizar entrevistas secuenciales con cada caso de estudio identificado.

\section{Resultados y Discusión}

\section{Proceso de crianza de camélidos sudamericanos domésticos}

El sistema de crianza de camélidos domésticos practicado por criadores implica el pastoreo trashumante y rotatorio, es decir, en temporada de estiaje, de abril a octubre, el rebaño de alpacas ${ }^{5}$ se traslada a estancias de la parte alta y, durante temporada de lluvia, de noviembre a marzo, se traslada a estancias o fundos ubicados en la parte baja, así como la rotación en una misma estancia. El traslado de una estancia a otra (trashumancia) 
y la rotación dentro de la misma estancia (rotatorio) cumplen una función en la prevención de mortandad de alpacas crías, recuperación y manejo de pastizales en fundos ganaderos, por tanto, el pastoreo trashumante y rotatorio está relacionado con el conocimiento de la naturaleza, cuya situación es interpretada explícitamente por criadores desde su propia cosmovisión. Al respecto "en Chalhuanca, en época de lluvia trasladamos a zonas más bajas y dejamos las zonas altas, porque cae mucha nevada, más fuertes lluvias, por eso las zonas bajas aprovechamos en época de lluvia, para proteger las crías y tener pasturas reservadas arriba para épocas de secas. En mi caso, tenemos terreno desde nuestros abuelos, pero en otros casos se alquilan", decía nuestro informante.

Contar con más de una estancia o fundo facilita el manejo apropiado de la crianza de alpacas, pudiendo acceder a pasturas en épocas de lluvia y de estiaje, por otro lado, tener una estancia, a veces con insuficiente extensión, va en detrimento de la producción y dificultades para mejorar la crianza de alpacas, siendo necesario alquilar fundos ganaderos. La crianza de alpacas, en escasos terrenos con pasturas insuficientes o bajo condominios de familias, ha tenido consecuencias muy serias, especialmente en familias pobres con una sola estancia o fundo, como sobrepastoreo, desertificación de suelos, baja productividad. El pastoreo y la mejora de pastos en estas zonas ha sido bastante estudiado (Flores 1977, 1990; Tapia y Flores 1984; Moya 1994), pero no es mucho lo que se avanzó por solucionar estos problemas considerando las alternativas locales. Algunas familias alpaqueras han estado realizando mejoras, recurriendo a sus prácticas y conocimientos locales (tecnología propia), cabe destacar el abonamiento de tierras, poner cercos, ampliación de bofedales mediante apertura de zanjas de filtración, conservación de pastos cultivados mediante la técnica del ensilaje de forraje ${ }^{6}$, para épocas de estiaje o nevada excesiva. Estas actividades implican la participación y cooperación de toda la familia, pero en muchos casos, solo las mujeres y sus hijos se quedan junto a las alpacas en los predios, dado que sus esposos salen a vender productos o en busca de trabajo. Una informante de Caylloma distrito señala:

En la crianza se dedican ambos, mujer y marido, aunque el marido raras veces viene a pastear, yo más vengo, solo cuando hay que trabajar por los hijos en la escuela no vengo, siempre las mujeres tenemos más preocupación en todo, el marido de repente se va al trabajo o a las minas, entonces estamos siempre las mujeres al lado de nuestras alpacas, hasta en la misma comercialización de fibra, porque a veces no están y ahí tenemos que estar nosotras...

La participación de la familia en la crianza de camélidos, liderada por mujeres, es valorada por las sociedades pastoriles, permite que ellas tengan iniciativa sobre el manejo de alpacas, manejo de pastos, comercialización, tratamiento de enfermedades que atacan a las alpacas como karachi (sarna), fiebre, diarrea, parásitos, jamak'o (garrapata) y otras. Los criadores señalan que los animales se quejan de sus dolencias, haciendo movimientos con la cabeza o retorciéndose, su tratamiento requiere de fármacos de laboratorio y de medicamento tradicional (yerbas y orín humano). Todavía en muchos casos se combina su aplicación para tratar enfermedades que padecen las alpacas, algunos ganaderos cuentan con pequeños botiquines, empero el uso de fármacos de laboratorio en exceso o exclusivamente, ha tenido efectos adversos en la sanidad de camélidos. El estudio realizado por Moya (1994) con alpaqueros aymaras, devela que la medicina veterinaria de laboratorio tiene efectos negativos como: enflaquecimiento y alteración de la carne por aplicación de Novalgina para fiebre, caída de fibra por aplicación de Emicina para sarna.

En muchos casos el uso de medicamento tradicional o casero resulta ser menos nocivo que los fármacos de laboratorio. La pervivencia hasta nuestros días del uso de medicina natural demuestra que los conocimientos y prácticas locales tienen utilidad en la crianza de camélidos. Estos conocimientos y prácticas locales, normalmente no son considerados ni promocionados por los programas de desarrollo, por el contrario incentivan el uso casi exclusivo de fármacos de laboratorio, dejando al olvido el uso de la medicina tradicional, siendo más económica, menos nociva ecológicamente, existe en la zona y está al alcance de los criadores. Son alternativas de prácticas culturales y ecológicas muy diferentes a la modernidad homogenizante, que responde a lógicas consumistas y a necesidades del mercado, mas no a necesidades del lugar ni a la diversidad cultural y ecológica. Es cierto que no podemos prescindir los medicamentos de laboratorio, pero su aplicación debe 
ser moderada y combinarse adecuadamente con la medicina tradicional o natural, lo que depende del tipo de enfermedad, por lo que su combinación es viable e intrínseca. Así lo señala nuestro informante de Chalhuanca (distrito Yanque):

Es necesario que siempre estemos utilizando ambas, la medicina casera a veces son mucho más efectivas que antibióticos, pero la ciencia ha avanzado, las plantas medicinales no pueden controlar todo, entonces, una mezcla se puede hacer, lo que estamos haciendo ahora, el campesino alpaquero de acuerdo a sus necesidades adapta a su tecnología, viendo cual es mejor para su hato ganadero.

De los siete distritos (Tuti, Sibayo, Callalli, Caylloma, Tisco, San Antonio de Chuca y Yanque) (Figura 1) dedicados a la crianza de camélidos, Caylloma, Callalli y Yanque (su anexo Chalhuanca) son distritos que destacan por tener mejores ejemplares en alpacas, donde se está trabajando en mejoramiento de fibra, sanidad, genética, empadre controlado, selección de reproductores, construcción de cobertizos, pastos cultivados, administración de fundos. Este proceso todavía es incipiente, se necesita ampliar la cobertura de servicios, poniendo énfasis en la comprensión y utilidad de sus conocimientos y prácticas, sus creencias y capacidades, aspectos que determinan su comportamiento y estilo de vida. Sin embargo, el conocimiento de la cultura [local] no implica instrumentalizarlo para ampliar un modelo de desarrollo exógeno, por el contrario, debe favorecer el desarrollo de la cultura local, es un fin en sí misma a alcanzar ${ }^{7}$, a la que el crecimiento económico y la innovación tecnológica deben contribuir.

El conocimiento local es un modo de conciencia, centrado en el lugar, una forma de dotar significado al mundo en que viven los criadores, pero en procesos de desarrollo [preocupados por la modernización y la globalización] lo han perdido de vista. En este contexto, el posdesarrollo es una herramienta heurística para valorar, aprender, ver y reexaminar la realidad de los pueblos, es una posibilidad para pensar pensamientos distintos y escribir en otros lenguajes [locales]. La meta del posdesarrollo (según Escobar 2000) es hacer visibles las prácticas sociales, políticas y económicas locales, para ello es necesario reconocer el lugar como cultura local y repensar la modernización y tecnificación en términos del lugar, ecológica y culturalmente diferenciado.

Desde esa perspectiva, los conocimientos, capacidades, creencias y prácticas locales son valiosos en la crianza de camélidos, al que todavía se recurre y orienta la cotidianidad de los criadores. Entonces, la crianza de camélidos no es una actividad aislada o puramente técnica, como muchas veces entienden los técnicos del desarrollo. Para los criadores es mucho más que una simple actividad, es un proceso holístico, donde convergen la naturaleza, la comunidad y lo sobrenatural, cuya interdependencia está determinada por prácticas, conocimientos y creencias, de modo que es importante entender la relación de los rituales con la crianza o comercialización desde una visión integral, todavía en las estancias o fundos alpaqueros se conserva su práctica, entre los más realizados están: ritual del tinkachi, pago a la tierra y señaje o marcación.

El ritual del tinkachi, realizado en carnavales, para festejar a las alpacas, durante la temporada de pariciones y empadre, de gran significado para los criadores. La parición significa la bendición de la naturaleza, expresado en la reciprocidad con los criadores. El empadre, no solo es un simple apareamiento de un macho con la hembra, es el encuentro comunitario entre la naturaleza, criador de camélidos y dioses, que garantizan la reproducción de la vida en general, en una ecología de la cultura local. Por otro lado, el ritual pago a la tierra, realizado en agosto (koñikilla), consiste en dar las gracias a la tierra dadora de vida. Según los criadores, la Santa Tierra habla, el pastor tiene que estar con su pito ${ }^{8} \mathrm{y}$ vino, junto a otras especies para ofrendar a la tierra. Por último, el ritual de señaje o marcación entre los aymaras (Moya 1994), realizado en fechas significativas como navidad, año nuevo, significa señalar una alpaca en nombre de sus hijos o de un conocido muy cercano a la familia, para que se reproduzca. En su lenguaje dicen "kay wata señaykusayki kay pakochata sutiykipi mirananpaq". A continuación algunos ritos realizados, que es explicado por dos informantes, una de Ran Ran (Tuti) con el tinkachi y el otro de Chalhuanca (Yanque) con pago a la tierra:

El tinkachi significa lo que una persona hace sus cumpleaños, así nosotros hacemos a nuestros animales, celebramos y tinkamos para que se reproduzca, nosotros seguimos haciéndolo. 
Hacemos rituales a recursos naturales como la mamaqocha y pachamama, mamaqocha le dicen a los manantiales, pachamama a la tierra donde están las alpacas y llamas. Había personas especialistas en hacer rituales, se llamaban los Laris (hombres) y las Hipas (mujeres). Se hace actualmente en agosto, los productores hacen pago a la tierra, para que les vaya bien todo el año, hacen pago a los Apus, cerros tutelares de los fundos, con una especie de sellado, se llama Apajata, que llevan como regalo y pagan a la tierra, generalmente los regalos son feto de llama, chicha, algunos frutos andinos como quinua, kañigua, flores, dulces, mezclados se queman en nombre de un cerro. También se mata un animal y se paga, se come como wilancho (sacrificio). Bueno, hace años pensaba que eso era un juego, pero me dado cuenta, realmente todo los recursos que existen en la naturaleza son una especie viva y comparten con nosotros, para mí es rescatable.

Este caso explica que la crianza de camélidos significa una relación de reciprocidad con la naturaleza y los dioses, por lo que una intervención social para mejorar o tecnificar la crianza de camélidos, debería entender e integrar esta noción holística culturalmente establecida en la comunidad local. Quizá la noción mejor establecida hoy en día en sociedades "modernas", es la que reposa sobre la dicotomía naturaleza/sociedad. Al contrario de construcciones conceptuales "modernas", con su separación estricta entre el mundo físico, biológico y espiritual, los modelos locales no occidentales se fundamentan sobre la continuidad, como un todo, determinado culturalmente a través de símbolos, rituales, prácticas y conocimientos, que se insertan en el tejido social de sociedades particulares [esto resulta incierto y problemático para los "otros"], siendo entendible desde una mirada de la cultura local. Las relaciones sociales abarcan más que humanos, en la cosmovisión del alpaquero confluye la naturaleza, la sociedad y las deidades, de modo que, seres vivientes, no vivientes y a menudo los sobrenaturales, no se consideran como dominios distintos y separados, eso echa por tierra la dicotomía señalada.

Comprender y tener como base la lógica del pensamiento andino [de criadores] en procesos de modernización es de importancia significativa, pero a menudo se olvida su realidad sociocultural, lo que deviene en fracasos o resultados pésimos, así lo demuestra Conrad Phillip (2000), al evaluar varios programas alrededor del mundo, que fracasaron al no prestar atención a la compatibilidad y la diversidad cultural (sobreinnovación e infradiferenciación), al usar más modelos de plantilla y menos modelos de aprendizaje. Poner atención a la diversidad cultural y a la cosmovisión local, ayuda a comprender que, los procesos de modernización local deben ser compatibles y ayudar al progreso de la cultura local.

\section{Proceso de comercialización practicada por criadores de camélidos}

En zonas altoandinas, desde tiempos precoloniales, el trueque o intercambio de mercancías entre pueblos, ha sido un componente vital en el sustento del hogar, facilitó el abastecimiento de productos agrícolas y frutas a familias dedicadas a la crianza de camélidos domésticos (Markowitz 2006). Sobre el particular, el criador de camélidos de Chalhuanca (Yanque), relata:

Hace poco todavía hacíamos trueque, llevábamos artesanía, charqui a Cabanaconde para truequear con maíz..., antes el trueque tenía un sistema de medición, era en costal, le llamábamos chimpu, se medía por cuartas, por ejemplo una pierna de alpaca para una cuarta o dos cuartas, era cuestión de hacer transacción comercial, en otras zonas era por tarro de aceite, un tarro lleno para una pierna. En artesanía utilizaban la frazada cosida en cuatro, te daban lo que llenaba a la frazada. Hoy en día vienen a la feria los comerciantes, traen todo lo que necesitamos, lo que hacemos ahora es vender, buscar buen precio para nuestros productos, con eso cubrir necesidades.

El debilitamiento del trueque va unido a la creciente articulación al mercado, con la ampliación de vías de comunicación hacia pueblos altoandinos, junto al establecimiento de ferias semanales en los distritos de la parte alta de la provincia de Caylloma. A finales de la década de 1980 se amplía el comercio de fibra en la provincia, que coincide con la ampliación de ferias semanales, hecho que intensifica el intercambio comercial en las sociedades 
pastoriles (Markowitz 2006:334). Estos antecedentes han configurado el panorama actual del proceso de comercialización de fibra, hoy existe en casi todo los distritos y anexos de la parte alta de Caylloma ferias semanales, donde productores, intermediarios y comerciantes venden y compran productos.

Los criadores venden productos como lana, fibra, carne, charqui, pieles (carcaza), artesanía (tejidos), pomayos (cuero con fibra de corte pequeño) y últimamente reproductores mejorados, obviamente se comercializa más la carne y fibra, mientras otros subproductos se vende según urgencias o necesidades de gasto, algunas familias utilizan como caja chica las pocas ovejas o llamas que disponen en su rebaño. Los productos emblemáticos como fibra y carne se comercializan según temporada. La carne se vende en campaña de saca junio a noviembre ${ }^{9}$, en cambio la fibra (sea de tipo sury o huacaya) se vende durante la campaña de acopio noviembre a marzo, es el tiempo de esquila. Esta producción es vendida a intermediarios en ferias locales y/o centro de acopio local, liderados por comités de acopio distrital.

La formación de Comités de Acopio local o distrital responde a la iniciativa de los criadores que buscan mejorar el precio a través de la categorización de fibra y comercialización asociada mediante Centros de Acopio. El Comité de Acopio de fibra es una organización formada por criadores, al mismo tiempo se constituye un Comité de Vigilancia, integrada habitualmente por autoridades locales y un representante de los criadores, que cumple la función de supervisar el proceso de acopio y comercialización. Los primeros centros de acopio de fibra empezaron en el año 2005 en Callalli y Chalhuanca (distrito Yanque), en años posteriores fueron ampliándose a diversas zonas alpaqueras de la provincia Caylloma (Toqra, Imata, Pachachaka, Kotakota, Qenko Q'ala Q'ala, Tisko, Chichas, Ichuhuayqo y Tuti). El acopio de fibra tiene sus inicios por la década de 1980 y 1990 del siglo pasado en Huancavelica, Arequipa y Puno (Ágreda 1997). El Proyecto CECI alpaca en Puno organizó a criadores formando empresas cooperativas para acopio y transformación de fibra destinada a la exportación, valorando los conocimientos locales (Yeckting 2006:156-159). La asociación ADECALC ${ }^{10}$, en Caylloma (Arequipa), acopió fibra entre sus socios alpaqueros. Son experiencias comerciales que demuestran la iniciativa local para la industrialización y la formación de clusters empresariales, sustentado en conocimientos y prácticas locales.
El [nuevo] sistema de comercialización asociada de fibra categorizada a través de centros de acopio, que establece precios diferenciados por calidad, todavía sigue siendo marginal. En el caso de la provincia de Caylloma llega a cubrir al 3\% de la producción de fibra y abarca al $6 \%$ del total de criadores (CONACS 2007). Significa que el sistema de comercialización a través de intermediarios es predominante, de seguir este sistema que no mejora los precios ni incentiva la mejora genética, el Perú pondría en riesgo la calidad de fibra requerida por el mercado mundial de pelos finos, dominado ahora por el Mohair y el Cashmere, de ahí la importancia de la comercialización asociada de fibra. En este proceso comercial, la industria clasifica la fibra según su diámetro en cinco niveles: royal, baby, médium flece, huarizo y gruesa corta, pero en Centros de Acopio de Caylloma se categoriza la fibra según finura en gruesa, semifina, fina y extrafina, por el contrario, con intermediarios no hay dicha categorización, pues realizan compras al "barrer", considerando solo dos categorías: fibra adulta y fibra cría o tuwi, ya sea del tipo suri (mary) o huacaya (wakaya) (Figura 2), esta última es la que más abunda en Caylloma.

En el Centro de Acopio de fibra del distrito Callalli, se observó una discusión entre un criador y el Comité de Acopio, por la categorización que recibió la fibra, el criador alegaba que su fibra era mejorada y en la categorización no calificó como mejorada. El desconocimiento de la calidad de fibra y la costumbre de entregar al "barrer" a intermediarios determinó el desacuerdo en la venta. Esto explica, en parte, por qué solo la tercera parte de criadores entregan fibra a centros de acopio, sumado a la desconfianza de entregar fibra con pago diferido. Los criadores son conscientes de que el proceso de comercialización asociada se debe seguir mejorando, dándoles un valor agregado a los productos, explicando los beneficios de venta asociada, motivando a que mejoren la calidad de fibra, sin descuidar la variedad de colores ${ }^{11}$ genéticamente determinados. Uno de los criadores de Callalli explica la ventaja de los centros de acopio de fibra:

Creo con acopio de fibra hay enorme ventaja, porque ganamos por doble partida, primero categorizando nuestra fibra, segundo por el precio que nos paga los centros de acopio. El intermediario solo compra en dos categorías, fibra adulta y fibra cría, 


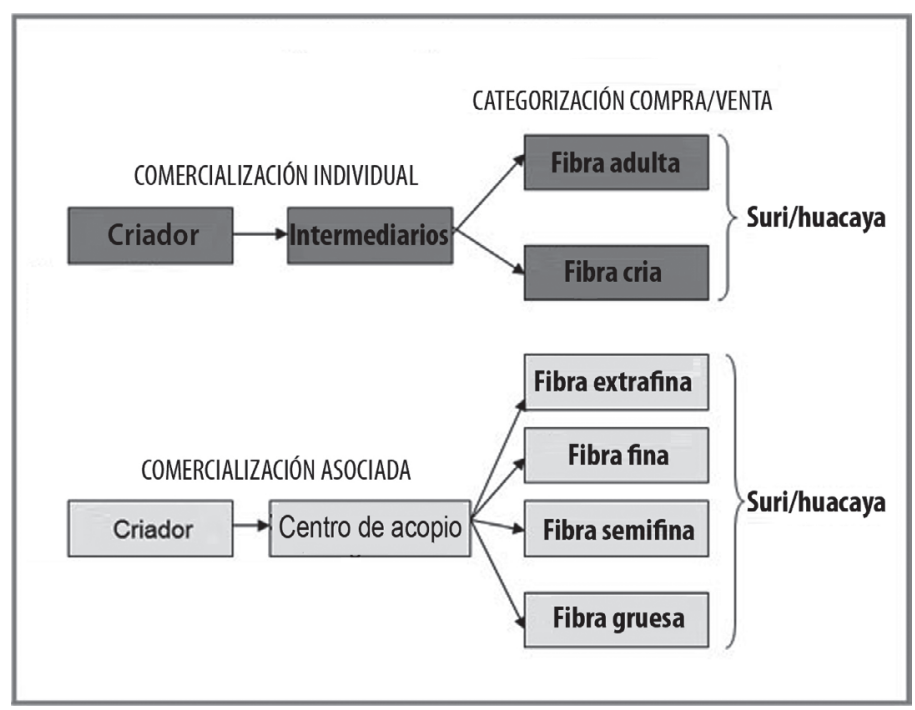

Figura 2. Categorización según sistema de comercialización. Categorization according to the system of commercialization.

pero dentro de fibra adulta, por el trabajo de mejoramiento que uno hace, siempre hay fibra fina, entonces, el intermediario aprovecha eso para vender a otro precio, ahí perdemos, otro es el peso, tienen mañas, saben cómo ganar y a quien agarrar.

Para los criadores la comercialización asociada de fibra y organizada mediante centro de acopio, es una iniciativa que no solo responde al deseo de mejorar los precios o distinguir la calidad de fibra, sino también a modelos de cooperación familiar, que subyace a formas de reciprocidad practicada en la cultura local. Markowitz (2006) sostiene que son esos modelos arraigados a su cultura, lo que les anima a participar en estrategias colectivas, donde el interés común trasciende a las diferencias de riqueza y posesión social. La cooperación no solo se manifiesta en la comercialización asociada, sino también en tareas como esquilar, dosificar animales, abrir zanjas de filtración, pastorear. Experiencias de este tipo demuestran que las estrategias colectivas, sustentadas en un conjunto de conocimientos, valores, creencias y prácticas distintivas (capital cultural), y niveles de asociatividad comunal, relaciones o redes sociales (capital social) son perfectamente viables en procesos de mejoramiento local implementados participativamente. La comercialización asociada de fibra ilustra la persistencia en sus valores y creencias propias, sobre la necesidad de organizarse y asociarse entre criadores. Esto no es otra cosa que las alianzas estratégicas en procesos de comercialización. Por tanto, es momento de revalorar y entender los conocimientos y creencias de las personas, de confiar en sus capacidades de organización y asociatividad en términos del lugar.

Estas prácticas comerciales locales, al igual que la crianza de alpacas, son un proceso global; para los ganaderos los rituales están presentes en la compra y venta. La vida económica que ellos entienden está basada en la mutua interdependencia con la comunidad, la naturaleza y lo sobrenatural, que garantizan su funcionamiento y reproducción, así como su interrelación con otras economías o modelos económicos culturales. Dicha interdependencia se materializa en los rituales realizados, los que todavía perviven son: ritual de esquila (al iniciar la esquila de fibra) y ritual de sahumada de fibra (al preparar la venta de fibra acopiada), en cuya práctica interviene chicha, coca, incienso y otros insumos. La realización del ritual es una expresión de su cosmovisión representado en el estilo de vida, que le otorga confianza y seguridad en las transacciones comerciales, es decir, desde la concepción de los criadores, su práctica propicia una buena comercialización. Un informante de Chalhuanca expone al respecto:

Al comprar una casa, recibir harta cantidad de dinero o vender, hacen su sahumerito, su challadita, para que todo salga bien. Bueno, 
en el primer acopio hemos challado con cerveza, hemos sahumado con incienso; el segundo acopio nos ha resultado un poco mal, no hemos hecho nada, el tercer acopio si han challado un poco, regular está...

Para una economía de mercado el comportamiento descrito puede ser considerado poco razonable o hasta ilógico, pero para la economía doméstica o rural, tiene lógica y correspondencia con su estilo económico. La antropología económica desde sus enfoques recientes de etnoeconomía o economía cultural (Bird-David 1997), contribuye a esclarecer este problema y dilucida que existen diversas conceptualizaciones sobre la lógica de comercialización, una forma cultural de pensar la economía, donde el conjunto de conductas o comportamientos se vuelven más razonables, que de otra manera parecerían ilógicas y curiosas. Desde esa perspectiva, la práctica de rituales es una parte importante del proceso de comercialización asociada de fibra que los criadores realizan, dichos rituales también son recreados por empresarios de la micro y pequeña empresa, conocidos como rituales empresariales (Arrosquipa 2002, 2011), cuya realización incide en la cohesión grupal (identidad corporativa), preparación de equipos de trabajo (trabajo en equipo), visión compartida (planificación), de ahí su importancia en la gestión empresarial o en procesos de comercialización asociada.

Estas formas de pensar la comercialización no son problema para que las personas participen en más de una economía y a menudo en más de una comunidad cultural, por el contrario, constituyen la base para llevar adelante procesos de desarrollo y modernización local, en crianza y comercialización. Una modernización que no propicie la desaparición de costumbres y conocimientos locales, sino que los revitalice y difunda.

\section{Consideraciones Finales}

En la crianza de camélidos domésticos (alpacas), comprender el pastoreo trashumante (parte baja y parte alta) y la rotación en un mismo fundo o estancia (manejo de pastizales), como parte intrínseca del proceso, ayuda a superar los desencuentros culturales y es vital para fortalecer capacidades locales, situación que lleva a sintonizar mejor los conocimientos y prácticas locales en procesos de modernización local de las comunidades.
En el proceso de crianza de camélidos y comercialización de fibra no existe la dicotomía naturaleza cultura, como mundos separados, distintos o aislados, sino es una continuidad cíclica culturalmente establecida, de la que depende su reproducción y continuidad, que los proyectos de desarrollo a menudo olvidan al intervenir en comunidades. Así, la cosmovisión holística de los criadores de camélidos es compatible con los postulados del desarrollo sostenible, allí radica la importancia de recuperar y difundir los conocimientos locales para la sostenibilidad económica, social y ambiental.

En transacciones comerciales que acontecen en ferias semanales de cada distrito, se desarrollan relaciones comerciales entre criadores y agentes externos, como el sistema de comercialización individualizada [a través de intermediarios] y sistema de comercialización asociada [a través de centros de acopio]. Sin embargo, el [nuevo] sistema de comercialización asociada de fibra ha ido tomando mayor relevancia, un modelo que responde a la cooperación familiar y reciprocidad practicada en la cultura local, donde la participación en estrategias colectivas trasciende a las diferencias económicas y sociales de las familias criadoras, constituyen aspectos fundamentales para promover la modernización local, mediante clúster empresariales en diferentes niveles de la cadena productiva, a partir del capital social y cultural existente entre los criadores.

Las prácticas y conocimientos locales desarrollados por criadores de camélidos, en procesos de crianza y comercialización, son esenciales y pertinentes. Por tanto, estas prácticas y conocimientos locales tienen viabilidad práctica y compatibilidad para ser recreados en procesos de modernización o desarrollo basado en la cultura local, cuya sostenibilidad halla sus raíces en prácticas y discursos alternativos sobre la diversidad cultural y ecológica.

Agradecimientos: Esta investigación fue realizada gracias a una beca otorgada por el Programa Jóvenes Investigadores SEPIA XII/Fundación Ford. Se agradece el respaldo institucional y logístico de la ONG ASDE (Acción Social y Desarrollo), a los criadores de camélidos de la provincia de Caylloma por su amabilidad, hospitalidad y permitirme participar de sus actividades cotidianas. A los evaluadores anónimos de Chungara por las sugerencias y precisiones. 


\section{Referencias Citadas}

Arrosquipa, P. 2002. La Cultura Empresarial: Una Visión Antropológica desde las Pymes. Tesis para optar la Licenciatura en Antropología, Facultad de Ciencias Histórico Sociales, Universidad Nacional de San Agustín, Arequipa.

- - - 2011. Cultura: prácticas y conocimientos de criadores de camélidos. Actas VII Congreso de la Asociación Latinoamericana de Especialistas en Pequeños Rumiantes y Camélidos Sudamericanos ALEPRyCS, editado por J. Ruiz y A. Cordero, pp. 387-391. Universidad Nacional de Huancavelica, Huancavelica.

Ágreda, V. 1997. La comercialización interna de la fibra de alpaca después del ajuste. En Perú: El Problema Agrario en Debate SEPIA VI, editado por E. Gonzáles de Olarte, B. Revesz y M. Tapia, pp. 173-202. SEPIA, Lima.

Bird-David, N. 1997. Las economías: una perspectiva económico cultural. Revista Internacional de Ciencias Sociales 154. UNESCO. http://www.unesco.org/issj/rics154/birdrspa.html

Baigorri, A. 2001. Modelos de desarrollo rural y sostenibilidad. http://www.unex.es/sociolog/BAIGORRI/papers/agroecologia. $p d f$ (15 marzo 2008).

CONACS 2007. Informe Comité de Coordinación Regional de Comercialización. CONACS, Arequipa.

DESCO 1985. Alpaqueros de Caylloma. Desco, Arequipa.

Escobar, A. 1997. Antropología y desarrollo. Revista Internacional de Ciencias Sociales 154. UNESCO. http://www.unesco.org/ issj/rics154/escobarspa.html

- - - 2000. El lugar de la naturaleza y la naturaleza del lugar: globalización o posdesarrollo. En Antropología del Desarrollo, editado por A. Viola, pp. 196-209. Paidos, España.

- - - 2005. El "postdesarrollo" como concepto y práctica social http://www.unc.edu/ aescobar/text/esp/El\%20postdesarrollo\%20 como\%20concepto.pdf (20 noviembre 2013).

Esteva, G. 2000. Desarrollo. En Antropología del Desarrollo, editado por A. Viola, pp. 67-100. Paidos, España.

Foster, G. 1976. Las Culturas Tradicionales y los Cambios Técnicos. Segunda reimpresión, Fondo de Cultura Económica, México.

Flores, J. (compil.) 1977. Pastores de Puna (Uywamichiq Punarunakuna). Primera edición, Instituto de Estudios Peruanos, Lima.

- - - 1990. Pastores Alto Andinos: Origen, Desarrollo y Situación Actual. Ceac, Qosqo.

FAO 2008. Análisis del Impacto de los Eventos Fríos (friaje) del 2008 en la Agricultura y Ganadería Alto Andina del Perú. FAO. Lima
Geertz, C. 1992. La Interpretación de las Culturas. Gedisa, España.

García, J.J. 1996. Racionalidad de la Cosmovisión Andina. Primera edición, Concytec, Lima.

Healy, K. 2002 [2001]. Llamas, weaving and organic chocolate, reseñado por Michael Foley, Desarrollo de Base 23/1. Inter Americana Fundation, Virginia.

INEI 2013. Resultados definitivos IV Censo Nacional Agropecuario 2012. INEI, Lima.

- - - 2007. Censo Nacional 2007: XI de Población y VI de Vivienda. INEI. Lima.

Markowitz, L. 2006. ¿Cómo ganarse la vida? Estrategias de los alpaqueros de Caylloma. En Camélidos Sudamericanos Domésticos, editado por C. Reniere, E. Frank y O. Toro, pp. 334355. Desco, Lima.

Moya, E. 1994. Cosmovisión y Conocimiento de los Alpaqueros Aymaras. INIAA, Lima.

Phillip K., C. 2000. La cultura y “el desarrollo económico”. En Antropología del Desarrollo, editado por A. Viola, pp. 103-125. Paidos, España.

Rist, G. 2000. La cultura y el capital social: ¿cómplices o víctimas del "desarrollo"? En Capital Social y Cultura: Claves Estratégicas Para el Desarrollo, compilado por B. Kliksberg y L. Tomassini, pp. 129-150. Primera edición, Fondo de Cultura Económica/Banco Interamericano de Desarrollo, Argentina.

Spedding, A. 1993. Semiótica de la cocina paceña andina. Unitas 10:51-64.

Tapia, M. y J. Flores 1984. Pastoreo y Pastizales de los Andes del Sur del Perú. INPA/PCIRM, Lima.

Urbano, E. (compil.) 1991. Modernidad en los andes. En Debates Andinos $\mathrm{N}^{\circ}$ 17. CERA Bartolomé de las Casas, Cusco.

Van Der Ploeg, J.D. 2000. Sistemas de conocimiento, metáfora y campo de interacción: el caso del cultivo de la patata en el altiplano peruano. En Antropología del Desarrollo, editado por A. Viola, pp. 359-382. Paidos, España.

Yeckting, F. 2006. La configuración de las visiones del desarrollo en el altiplano. La cooperación, desarrollo rural y el cambio técnico a través de los proyectos de desarrollo en las comunidades agropastoriles (1980-1990). En Perú: El problema Agrario en Debate SEPIA XI, editado por J. Iguiñiz, J. Escobal y C.I. Degregori, pp. 133-174. SEPIA, Lima.

Wheeler, J. 1991. Origen, evolución y status actual. En Avances y Perspectivas del Conocimiento de los Camélidos Sudamericanos. FAO, Santiago.

\section{Notas}

1 Las tendencias actuales de modelos de desarrollo rural tienen sus antecedentes en propuestas como el modelo de desarrollo propio que proponía Nyerere, consciente de la locura que supondría perseguir objetivos sentados por otros. El modelo del etnodesarrollo propuesto por Rodolfo
Stavenhagen, consciente de la necesidad de "mirar hacia dentro", buscar en la propia cultura en lugar de visiones prestadas o foráneas. El desarrollo de abajo arriba, sugerido por Jimoh Omo-Fadaka, consiente del fracaso de estrategias diseñadas desde arriba al no alcanzar sus objetivos. El 
desarrollo participativo en la que insistía Orlando Fals Borda y Anisur Rahman, ante la exclusión consumada en nombre del desarrollo. La propuesta de otro desarrollo para Japón de Jun Nishikawa, consciente de que la era actual estaba llegando a su fin, son propuestas que encuentran apoyo en "sociedades marginales" [del mundo económico], que viven según sus propias concepciones, recreado en barrios, aldeas y pueblos en todo el mundo, donde presupuestos no económicos gobiernan la vida y la economía ocupa un lugar marginal (Esteva 2000).

2 El término posdesarrollo se utilizó en 1991 por vez primera en un coloquio celebrado en Ginebra. Posdesarrollo es una manera de visualizar, concebir y construir estilos de vida de acuerdo con lógicas culturales propias del lugar. Es la reconstrucción del mundo desde la perspectiva de prácticas y discursos alternativos, basado en la autonomía y diversidad cultural y ecológica, dándole su sentido propio (identidad étnica). Según Escobar es la desconstrucción del desarrollo (Escobar 2005:3).

3 Son familias dedicadas a la crianza de camélidos domésticos, preferentemente de alpacas. La mayoría de las familias alpaqueras cuentan con más de un fundo ganadero, donde los rebaños de alpacas habitualmente son conducidos por las esposas, apoyado por abuelos y los hijos, quienes los fines de semana, después de sus labores escolares, vuelven al predio rural para apoyar con las actividades. Algunas familias alpaqueras (con rebaño de más de 500 alpacas) tienen pastores (personas huaqchos o pobres llamados huaqchilleros), que son contratados por temporadas. En el Perú 150 mil familias se dedican a la crianza de alpacas (FAO 2008).

4 Predio de la zona altoandina, dedicado a la crianza de camélidos preferentemente.

5 Los rebaños son de alpacas esencialmente, constituida preferentemente por raza huacaya y en menor proporción por suri, en algunos otros casos tienen pocas llamas y ovinos. Los rebaños en la mayoría de casos tienen en promedio 200 alpacas. Según último Censo Agropecuario del 2012, el Perú tiene 3.685 .516 alpacas, siendo el $80 \%$ de raza huacaya, de los cuales 468.392 alpacas están en la zona altoandina de Arequipa.

6 Proceso que comprende el empacado de forraje en silos construidos.

7 En procesos de desarrollo, el problema es la compatibilidad cultural de las realidades locales con el "desarrollo" en sí, más aun si esta se sigue entendiendo en términos económicos de países industrializados. Ante esta situación han tratado de "sumarle" cultura o capital social al desarrollo, dando muestra (dice Rist) de una gran ingenuidad o bien de un profundo cinismo y, sobre todo, de un extraño desconocimiento de antropología económica. Una discusión al respecto y de los arreglos cosméticos al desarrollo está en Rist (2000).

8 Chicha y maíz molido mezclado que se rosea al animal, según Peter Gose (1986, citado por Spedding) el pito es una bebida, que se hace mezclando la harina de maíz tostada en chicha, hasta que llegue a la consistencia. Spedding, también señala que el Wilancho es un sacrificio de la llama para fiestas o rituales, practicada en el mundo andino (Spedding 1993).

9 Temporada en la cual se sacrifican animales que culminan su ciclo o presentan algún defecto genético, cuyo índice productivo de carne en promedio es 20 a 30 kilos/alpaca y la fibra es 3 a 6 libras/alpaca/año (FAO 2008).

10 Asociación de Criadores Alpaqueros de Caylloma (ADECALC) que inició a finales de la década de 1980 acopiar fibra de socios alpaqueros y se descapitalizó a fines de 1990, diferente a los centros de acopio, donde la modalidad de entrega de fibra es libre y el acopio pública, con participación y/o apoyo de autoridades locales e instituciones de desarrollo público y privado.

11 Existen colores de fibra como: millo (LF color vicuña), yana (negro), chumpi (café) y chumpi oke (CEFE rojo), representa la variedad [genética] de colores en fibra, sea de raza huacaya o suri. 